\title{
Self-rated health as a comprehensive indicator of lifestyle-related health status
}

\author{
Chizumi Yamada $\cdot$ Kengo Moriyama $\cdot$ \\ Eiko Takahashi
}

Received: 12 October 2011/Accepted: 27 February 2012/Published online: 17 March 2012

(c) The Japanese Society for Hygiene 2012

\begin{abstract}
Objectives To evaluate the usefulness of self-rated health (SRH) as a comprehensive indicator of lifestyle-related health status by examining the relationships between SRH and: (1) history of cancer and cardiovascular disease; (2) treatment of hypertension, diabetes, and dyslipidemia; (3) abnormalities in clinical parameters including blood pressure, fasting glucose, and lipids; and (4) lifestyle habits.

Methods 3744 health-check examinees at Tokai University Hachioji Hospital seen between April 2009 and March 2010 were enrolled. SRH was graded as "good," "relatively good," "relatively poor," or "poor." For statistical comparison, the differences among "healthy" (=good), "relatively healthy" (=relatively good), and "unhealthy" (=relatively poor plus poor) groups were examined. Mantel-Haenszel odds ratios were calculated to remove the confounding effect of age, using the healthy group as the reference. The Mantel-extension method was used as a trend test.

Results 1049 subjects rated their health as good, 2194 as relatively good, 428 as relatively poor, and 73 as poor. The prevalence of all diseases showed significant odds ratios and trends as SRH deteriorated. Obesity, blood pressure,
\end{abstract}

Electronic supplementary material The online version of this article (doi:10.1007/s12199-012-0274-x) contains supplementary material, which is available to authorized users.

C. Yamada $\cdot$ K. Moriyama $\cdot$ E. Takahashi

Department of Clinical Health Science,

Tokai University School of Medicine, Tokyo, Japan

E. Takahashi $(\bowtie)$

Health Evaluation and Promotion Center,

Tokai University Hachioji Hospital,

1838 Ishikawa-machi, Hachioji, Tokyo 192-0032, Japan

e-mail: etaka@tokai.ac.jp glucose metabolism, and lipids deteriorated significantly as SRH became poorer, and a trend was observed in all parameters. Weight change, exercise, smoking, and rest showed significant odds ratios and trends as SRH deteriorated.

Conclusion SRH appears useful as a comprehensive indicator of lifestyle-related health status.

Keywords Self-rated health · Lifestyle-related disease . Health status · Indicator · Health examination

\section{Introduction}

There are various health indicators, including mortality, morbidity, medical examination abnormalities, lifestyle habits, medical expenses, activities of daily living (ADL), and quality of life (QOL). For health-check examinees in Japan, morbidity, medical examination abnormalities, and lifestyle habits are often used to evaluate their health status. However, the combined use of multiple indicators sometimes makes it difficult to assess their overall health. It would be of great use for not only individual but also populational health management if a comprehensive indicator of lifestyle-related health status were to be identified.

Self-rated health (SRH) is a single health measure based on subjective assessment of health status, and it has been preferentially used in social science research. SRH serves as an independent predictor of mortality, even after controlling for age, sex, and other demographic variables. Poor SRH is significantly related to increased mortality due to cardiovascular disease and cancer [1]. It has been reported that the causes of death that show a strong association with SRH are diabetes, infectious and respiratory diseases, and multiple causes, while heart disease, stroke, and cancer 
have a moderately strong association with SRH [2]. However, there have been few reports on the relationships between SRH and objective health indicators such as the prevalence of lifestyle-related diseases and abnormalities in clinical parameters.

In the present study, the usefulness of SRH as a comprehensive indicator of lifestyle-related health status was evaluated by examining the relationships between SRH and: (1) history of cancer and cardiovascular disease; (2) treatment of hypertension, diabetes, and dyslipidemia; (3) abnormalities in clinical parameters including blood pressure, fasting glucose, and lipids; and (4) lifestyle habits.

\section{Methods}

\section{Study population}

A total of 3744 subjects aged $\geq 20$ years ( 2074 men and 1670 women, mean age $50.7 \pm 11.9$ years) who underwent health examinations at Tokai University Hachioji Hospital between April 2009 and March 2010 was sequentially enrolled in this study. Verbal consent was obtained from the subjects to use their health records for analysis. The present study was cross-sectional in design and was approved by The Ethics Committee of Tokai University School of Medicine and complied with the Helsinki Declaration.

\section{Questionnaire survey}

SRH was assessed by asking "How do you feel about your health status?" using a self-administered questionnaire, and graded into one of four categories: "good," "relatively good," "relatively poor," and "poor." SRH was included in the questionnaire for routine health-check examinations, and all the subjects answered the questionnaire. Medical history was surveyed using self-administered questionnaires and interviews by nurses. The subjects with a history of cancer included those who had a present (currently treated or regularly followed-up) and/or past history of cancer. The other medical history and lifestyle habits were surveyed based on the questionnaire developed for a specific health examination and health guidance [3] (Supplementary Table 1). Alcohol consumption was surveyed by asking how many units of sake were drunk in a day, with 1 unit $(180 \mathrm{ml})$ of sake considered equal to $25 \mathrm{~g}$ of alcohol. Undesirable lifestyle habits were regarded as risks.

\section{Measurements}

Anthropometric measurements and blood sampling were carried out after overnight fasting. All measurements were included in the routine health-check examinations. Waist circumference (WC) was assessed at the end of expiration, measuring the minimum circumference at the level of the umbilicus to the nearest $0.1 \mathrm{~cm}$. Blood pressure of the right upper arm was measured with the subject seated. Fasting serum immunoreactive insulin (IRI) was measured by fluorescence-enzyme immunoassay (ST AIA-PACK IRI; Toso, Tokyo, Japan). Homeostasis model assessment of insulin resistance (HOMA-IR) was calculated as: fasting plasma glucose $(\mathrm{FPG})($ in $\mathrm{mg} / \mathrm{dl}) \times \mathrm{IRI}($ in $\mu \mathrm{U} / \mathrm{ml}) / 405$ [4] HOMA-IR $\geq 2.5$ was used to identify insulin resistance in this study, according to the reference interval of HOMA-IR which we recently determined following the stringent $\mathrm{C} 28$ A3 document from the Clinical and Laboratory Standards Institute using 2153 healthy Japanese subjects [5]. Serum lipid levels were measured enzymatically. Liver enzymes were measured following the standardized procedure of the Japan Society of Clinical Chemistry. The presence of fatty liver was detected by ultrasonography.

\section{Statistics}

Data are expressed as mean \pm SD. SPSS Statistics (version 19.0; SPSS Inc., Chicago, IL, USA) was used for the statistical analyses. For statistical comparison, the differences among "healthy" (=good), "relatively healthy" (=relatively good), and "unhealthy" (=relatively poor plus poor) groups were examined, because very few subjects rated their health as poor. Statistical significance for comparisons of clinical parameters among the groups was determined using Bonferroni's multiple-comparison tests. If the values showed no interaction with age, analysis of covariance was performed with Bonferroni's post hoc test including age as a covariate. The odds ratios and $95 \%$ confidence intervals for the "relatively healthy" and "unhealthy" groups were calculated using the "healthy" group as the reference. Mantel-Haenszel odds ratios were calculated to remove the confounding effect of age. The Mantel-extension method was used as a trend test [6]. All $p$ values were two-tailed, and $p<0.05$ was considered significant.

\section{Results}

Prevalence of lifestyle-related diseases

A total of 1049 subjects $(28.0 \%)$ rated their health as good, 2194 subjects $(58.6 \%)$ as relatively good, 428 subjects $(11.5 \%)$ as relatively poor, and 73 subjects $(1.9 \%)$ as poor. The clinical characteristics of the "healthy" (=good), "relatively healthy" (=relatively good), and "unhealthy" (=relatively poor plus poor) groups were compared. 
The prevalence of diseases by SRH status with age adjustment is presented in Table 1. There were no subjects with chronic renal failure. A significant increase in the prevalence of all diseases was associated with a lower $\mathrm{SRH}$, and a trend was observed as SRH deteriorated.

\section{Abnormalities in clinical parameters}

Table 2 presents the laboratory data of the three groups by SRH status. There was no difference in the male percentage among the groups: 603 men $(57.5 \%)$ in the healthy group, 1190 men $(54.2 \%)$ in the relatively healthy group, and 240 men $(55.9 \%)$ in the unhealthy group. The average age was significantly higher in the unhealthy group, followed by the relatively healthy group and the healthy group. FPG, HOMA-IR, and high-density lipoprotein cholesterol (HDL-C) showed no interaction with age, and were compared adjusted by age. As SRH deteriorated, WC, FPG, HOMA-IR, triglycerides (TG), and alanine aminotransferase (ALT) increased significantly, and HDL-C decreased significantly. Blood pressure and low-density lipoprotein cholesterol (LDL-C) were significantly increased in the relatively healthy and unhealthy groups compared with the healthy group. Body mass index (BMI)

Table 1 Disease prevalence by self-rated health status (3744 subjects who underwent health examinations at Tokai University Hachioji Hospital between April 2009 and March 2010)

\begin{tabular}{|c|c|c|c|c|c|c|c|c|}
\hline & \multirow{2}{*}{$\begin{array}{l}\text { Healthy } \\
n(\%)\end{array}$} & \multicolumn{3}{|c|}{ Relatively healthy } & \multicolumn{3}{|c|}{ Unhealthy } & \multirow{2}{*}{$\begin{array}{l}\text { Trend } \\
p\end{array}$} \\
\hline & & $n(\%)$ & OR & $95 \% \mathrm{CI}$ & $n(\%)$ & $O R$ & $95 \% \mathrm{CI}$ & \\
\hline Cancer $^{\mathrm{a}}$ & $12(1)$ & $73(3)$ & 2.70 & $1.50-4.88$ & $27(5)$ & 3.93 & $2.08-7.42$ & $<0.001$ \\
\hline Stroke $^{\mathrm{b}}$ & $10(1)$ & $62(3)$ & 2.77 & $1.44-5.34$ & $26(5)$ & 4.83 & $2.40-9.71$ & $<0.001$ \\
\hline Coronary heart disease $^{\mathrm{b}}$ & $17(2)$ & $81(4)$ & 2.14 & $1.27-3.60$ & $57(11)$ & 6.77 & $4.09-11.21$ & $<0.001$ \\
\hline Hypertension $^{\mathrm{c}}$ & $66(6)$ & $320(15)$ & 2.40 & $1.81-3.18$ & $124(25)$ & 4.38 & $3.18-6.05$ & $<0.001$ \\
\hline Diabetes $^{\mathrm{c}}$ & $10(1)$ & $73(3)$ & 3.29 & $2.82-10.28$ & $31(6)$ & 5.38 & $2.82-10.28$ & $<0.001$ \\
\hline Dyslipidemia $^{\mathrm{c}}$ & $60(6)$ & $194(9)$ & 1.45 & $1.06-1.97$ & $67(13)$ & 2.09 & $1.45-3.01$ & $<0.001$ \\
\hline
\end{tabular}

The odds ratios were age-adjusted by Mantel-Haenszel method. The Mantel-extension method was used as a trend test $O R$ odds ratio, $C I$ confidence interval

${ }^{a}$ Present and past history

b Past history

${ }^{c}$ Currently on treatment

Table 2 Subjects' laboratory data by self-rated health status (3744 subjects who underwent health examinations at Tokai University Hachioji Hospital between April 2009 and March 2010)

\begin{tabular}{|c|c|c|c|}
\hline & Healthy & Relatively healthy & Unhealthy \\
\hline Age (years) & $48.9 \pm 11.9$ & $51.1 \pm 11.6^{* *}$ & $53.1 \pm 12.4^{* *}, \# \#$ \\
\hline BMI $\left(\mathrm{kg} / \mathrm{m}^{2}\right)$ & $22.6 \pm 2.9$ & $22.9 \pm 3.3$ & $23.3 \pm 4.0^{* *}, \#$ \\
\hline $\mathrm{WC}(\mathrm{cm})$ & $81.3 \pm 8.7$ & $82.6 \pm 9.1 * *$ & $83.9 \pm 10.7 * *, \#$ \\
\hline $\mathrm{SBP}(\mathrm{mmHg})$ & $114.7 \pm 16.6$ & $117.4 \pm 16.8^{* *}$ & $118.9 \pm 17.6 * *$ \\
\hline DBP (mmHg) & $72.2 \pm 12.0$ & $73.9 \pm 12.2 * *$ & $74.6 \pm 13.0^{* *}$ \\
\hline FPG (mg/dl) & $97.9 \pm 12.3$ & $101.5 \pm 17.2 * *$ & $104.8 \pm 23.0^{* *}, \#$ \\
\hline HOMA-IR & $1.36 \pm 0.94$ & $1.62 \pm 1.25^{* *}$ & $2.03 \pm 2.14 * *, \# \#$ \\
\hline LDL-C (mg/dl) & $119.0 \pm 30.8$ & $123.7 \pm 31.6^{* *}$ & $123.3 \pm 31.2^{*}$ \\
\hline HDL-C (mg/dl) & $65.3 \pm 16.2$ & $63.6 \pm 16.4^{*}$ & $61.4 \pm 16.8^{* *}, \#$ \\
\hline TG (mg/dl) & $94.8 \pm 58.4$ & $104.9 \pm 69.7 * *$ & $115.3 \pm 73.0^{* *}, \#$ \\
\hline ALT (IU/l) & $20.7 \pm 12.4$ & $23.4 \pm 17.3 * *$ & $26.2 \pm 20.4 * *, \#$ \\
\hline$\gamma$-GT (IU/l) & $33.0 \pm 42.8$ & $37.3 \pm 51.7$ & $40.1 \pm 47.8^{*}$ \\
\hline
\end{tabular}

$S B P$ systolic blood pressure, $D B P$ diastolic blood pressure

Data are mean $\pm \mathrm{SD}$

Statistical significance was determined using Bonferroni's multiple-comparison tests. FPG, HOMA-IR, and HDL-C were compared adjusted by age

$* p<0.05, * * p<0.01$ compared with the healthy group

${ }^{\#} p<0.05,{ }^{\# \#} p<0.01$ compared with the relatively healthy group 
Table 3 Clinical parameter abnormalities by self-rated health status (3744 subjects who underwent health examinations at Tokai University Hachioji Hospital between April 2009 and March 2010)

\begin{tabular}{|c|c|c|c|c|c|c|c|c|}
\hline & \multirow{2}{*}{$\begin{array}{l}\text { Healthy } \\
n(\%)\end{array}$} & \multicolumn{3}{|c|}{ Relatively healthy } & \multicolumn{3}{|c|}{ Unhealthy } & \multirow{2}{*}{$\begin{array}{l}\text { Trend } \\
p\end{array}$} \\
\hline & & $n(\%)$ & $O R$ & $95 \% \mathrm{CI}$ & $n(\%)$ & $O R$ & $95 \%$ CI & \\
\hline $\mathrm{BMI} \geq 25 \mathrm{~kg} / \mathrm{m}^{2}$ & $209(20)$ & $509(23)$ & 1.20 & $1.00-1.43$ & $154(31)$ & 1.76 & $1.38-2.24$ & $<0.001$ \\
\hline WC abnormality ${ }^{a}$ & $300(29)$ & $721(33)$ & 1.17 & $0.99-1.38$ & $186(37)$ & 1.37 & $1.09-1.71$ & $<0.01$ \\
\hline $\mathrm{SBP} \geq 130 \mathrm{mmHg}$ & $191(9)$ & $512(23)$ & 1.23 & $1.01-1.59$ & $141(28)$ & 1.47 & $1.14-1.90$ & $<0.005$ \\
\hline $\mathrm{DBP} \geq 85 \mathrm{mmHg}$ & $158(15)$ & $424(19)$ & 1.24 & $1.01-1.51$ & $107(21)$ & 1.34 & $1.02-1.76$ & $<0.05$ \\
\hline Hypertension $^{\mathrm{b}}$ & $234(22)$ & $627(29)$ & 1.26 & $1.05-1.51$ & $168(33)$ & 1.48 & $1.16-1.88$ & $<0.005$ \\
\hline $\mathrm{FPG} \geq 100 \mathrm{mg} / \mathrm{dl}$ & $375(36)$ & $961(44)$ & 1.31 & $1.12-1.53$ & $237(47)$ & 1.41 & $1.12-1.77$ & $<0.001$ \\
\hline HOMA-IR $\geq 2.5$ & $96(9)$ & $346(16)$ & 1.80 & $1.42-2.28$ & $119(24)$ & 2.91 & $2.19-3.87$ & $<0.001$ \\
\hline LDL-C $\geq 140 \mathrm{mg} / \mathrm{dl}$ & $245(23)$ & $636(29)$ & 1.24 & $1.04-1.48$ & $137(27)$ & 1.10 & $0.86-1.40$ & NS \\
\hline HDL-C $<40 \mathrm{mg} / \mathrm{dl}$ & $36(3)$ & $91(4)$ & 1.21 & $0.82-1.79$ & $35(7)$ & 2.02 & $1.27-3.22$ & $<0.01$ \\
\hline $\mathrm{TG} \geq 150 \mathrm{mg} / \mathrm{dl}$ & $137(13)$ & $368(17)$ & 1.29 & $1.05-1.60$ & $107(21)$ & 1.74 & $1.33-2.30$ & $<0.001$ \\
\hline $\mathrm{ALT} \geq 31 \mathrm{U} / 1$ & $140(13)$ & 395 (18) & 1.46 & $1.18-1.79$ & 119 (24) & 2.07 & $1.58-2.71$ & $<0.001$ \\
\hline$\gamma-\mathrm{GT} \geq 51 \mathrm{U} / 1$ & $134(13)$ & 388 (18) & 1.42 & $1.15-1.76$ & 109 (22) & 1.85 & $1.40-2.44$ & $<0.001$ \\
\hline Fatty liver & $294(28)$ & 737 (34) & 1.25 & $1.06-1.47$ & $207(41)$ & 1.64 & $1.32-2.05$ & $<0.001$ \\
\hline
\end{tabular}

The odds ratios were age-adjusted by Mantel-Haenszel method. The Mantel-extension method was used as a trend test

$O R$ odds ratio, $C I$ confidence interval

a The cutoff values of $\mathrm{WC}$ were $\geq 85 \mathrm{~cm}$ for men and $\geq 90 \mathrm{~cm}$ for women

b Hypertension is defined as $\mathrm{SBP} \geq 130 \mathrm{mmHg}$ and/or DBP $\geq 85 \mathrm{mmHg}$

and gamma-glutamyl transpeptidase $(\gamma-\mathrm{GT})$ were significantly higher in the unhealthy group than in the healthy group (Table 3).

Abnormalities in clinical parameters by SRH status with age adjustment are shown in Table 4. For obesity, blood pressure, glucose metabolism, and liver enzymes, significantly higher odds ratios were observed in the relatively healthy and unhealthy groups compared with the healthy group. For lipids, the odds ratios were significantly higher except for LDL-C in the unhealthy group and HDL-C in the relatively healthy group. In addition, a trend was observed in which deterioration of all parameters was associated with lower SRH.

\section{Lifestyle habits by SRH status}

The undesirable lifestyle habits regarded as risks are presented in Table 4. For weight change, exercise, smoking, and rest, the odds ratios were significantly higher in the relatively healthy and unhealthy groups compared with the healthy group; a trend was observed in which deterioration of all lifestyle habits was associated with lower SRH. Of the questions on dietary habits, significant odds ratios and trends were observed regarding "skipping breakfast" and "snacking after supper." On the other hand, odds ratios and trends were not significant for "eating supper within $2 \mathrm{~h}$ before bedtime" and "fast eater." Further, the odds ratio and trend for alcohol consumption $\geq 75 \mathrm{~g}$ were not significant.

\section{Discussion}

This study aimed to evaluate the usefulness of SRH as a comprehensive indicator of lifestyle-related health status by examining the relationships between SRH and: (1) history of cancer and cardiovascular disease; (2) treatment of hypertension, diabetes, and dyslipidemia; (3) abnormalities in clinical parameters including blood pressure, fasting glucose, and lipids; and (4) lifestyle habits. As a result, significant increases in the prevalences of cancer and cardiovascular disease, treatment of lifestyle-related diseases, and abnormalities in clinical parameters were associated with lower SRH, and a trend was observed as SRH deteriorated. Some of the lifestyle habits, including weight change, exercise, smoking, and rest, showed significant associations with SRH.

SRH is based on a subjective assessment of health status and has been preferentially used as an alternative health measure in social science research when objective health indicators by medical examinations are unavailable. A comprehensive survey of living conditions by the Ministry of Health, Labor, and Welfare, Japan has been conducted every year since 1986 to investigate basic living conditions such as health, medical care, welfare, pensions, and income. The survey questionnaire contains a question on SRH. The distribution of SRH status in the present study was $28.0 \%$ good, $58.6 \%$ relatively good, $11.5 \%$ relatively poor, and $1.9 \%$ poor, which was similar to the result of the national survey in $2010(33.2 \%$ good, $53.0 \%$ 
Table 4 Undesirable lifestyle habits regarded as risks by self-rated health status (3744 subjects who underwent health examinations at Tokai University Hachioji Hospital between April 2009 and March 2010)

\begin{tabular}{|c|c|c|c|c|c|c|c|c|}
\hline & \multirow{2}{*}{$\begin{array}{l}\text { Healthy } \\
n(\%)\end{array}$} & \multicolumn{3}{|c|}{ Relatively healthy } & \multicolumn{3}{|l|}{ Unhealthy } & \multirow{2}{*}{$\begin{array}{l}\text { Trend } \\
p\end{array}$} \\
\hline & & $n(\%)$ & OR & $95 \% \mathrm{CI}$ & $n(\%)$ & $O R$ & $95 \% \mathrm{CI}$ & \\
\hline Weight gain $\geq 10 \mathrm{~kg}$ & $340(32)$ & $826(38)$ & 1.26 & $1.08-1.47$ & $224(45)$ & 1.69 & $1.36-2.10$ & $<0.001$ \\
\hline Weight change $\geq 3 \mathrm{~kg}$ & $259(25)$ & $617(28)$ & 1.19 & $1.01-1.41$ & $213(43)$ & 2.26 & $1.80-2.83$ & $<0.001$ \\
\hline Exercise $<2$ times/week & $710(68)$ & $1677(76)$ & 1.55 & $1.32-1.82$ & $425(85)$ & 2.67 & $2.03-3.52$ & $<0.001$ \\
\hline Physical activity $<1 \mathrm{~h} /$ day & $578(53)$ & $1392(63)$ & 1.41 & $1.22-1.64$ & $338(67)$ & 1.69 & $1.35-2.11$ & $<0.001$ \\
\hline Not fast walker & $390(37)$ & $995(45)$ & 1.40 & $1.21-1.63$ & $298(59)$ & 2.48 & $1.99-3.08$ & $<0.001$ \\
\hline Skipping breakfast & $117(11)$ & $361(16)$ & 1.57 & $1.26-1.96$ & $94(19)$ & 1.84 & $1.37-2.47$ & $<0.001$ \\
\hline Snacking after supper & $142(14)$ & $342(16)$ & 1.18 & $0.95-1.46$ & $89(18)$ & 1.38 & $1.03-1.84$ & $<0.05$ \\
\hline Supper $<2 \mathrm{~h}$ before bedtime & $298(28)$ & $649(30)$ & 1.06 & $0.90-1.25$ & $166(33)$ & 1.25 & $0.99-1.57$ & NS \\
\hline Fast eater & $370(35)$ & $808(37)$ & 1.07 & $0.92-1.25$ & $198(40)$ & 1.20 & $0.96-1.49$ & NS \\
\hline Alcohol consumption $\geq 75 \mathrm{~g} /$ day & $39(4)$ & $93(4)$ & 1.15 & $0.78-1.68$ & $28(6)$ & 1.53 & $0.93-2.52$ & NS \\
\hline Current regular smoking & $170(16)$ & $444(20)$ & 1.31 & $1.08-1.59$ & $116(22)$ & 1.56 & $1.20-2.03$ & $<0.001$ \\
\hline Insufficient rest & $292(28)$ & $960(44)$ & 2.02 & $1.72-2.36$ & $303(60)$ & 3.97 & $3.17-4.96$ & $<0.001$ \\
\hline
\end{tabular}

The Mantel-extension method was used as a trend test

$O R$ odds ratio, $C I$ confidence interval

moderate, $12.4 \%$ relatively poor, and $1.4 \%$ poor; aged 45-54 years) [7].

For health-check examinees, there are various health indicators, such as morbidity, medical examination abnormalities, and lifestyle habits. However, the combined use of multiple indicators sometimes makes it difficult to assess their overall health; For example, it is difficult to determine the health status of an individual "who has elevated blood pressure, decreased blood glucose, increased LDL-C, but reduced TG" who changes his/her lifestyle habits to "start exercise, but increase the amount of drinking and eat supper within $2 \mathrm{~h}$ before bedtime," because there are mixed cases of deterioration and improvement in both clinical parameters and lifestyle habits. Therefore, a single health measure is needed to comprehensively assess various indicators, and the usefulness of SRH as a comprehensive indicator was evaluated in the present study.

In the early studies of SRH conducted in the USA in the 1950s and 1970s [8-10], it was reported that SRH was statistically associated with objective health indicators, though its validity was too low to be used as an alternative for medical diagnosis. Nevertheless, it has been suggested that another aspect of health may be measured by SRH, and SRH is expected to be a comprehensive health indicator. The extreme of poor health is "death," and there have been many reports on the relationship between SRH and mortality. Mitoku et al. [1] reviewed more than 40 studies and reported that SRH serves as an independent predictor of mortality, even after controlling for age, sex, and other demographic variables. In addition, poor SRH is significantly related to increased mortality due to cardiovascular disease and cancer, and the impact of SRH differs by cause of death.
Benjamins et al. [2] examined the relationship between SRH and mortality and reported that the causes of death that show a strong association with SRH are diabetes, infectious and respiratory diseases, and multiple causes, while heart disease, stroke, and cancer have a moderately strong association with SRH, and deaths due to accident, homicide, and suicide have a weak or no association with SRH.

As the population rapidly grows older in Japan, the distribution of disease has changed. The proportion of lifestyle-related diseases such as cancer, coronary heart disease, cerebrovascular disease, and diabetes is increasing, accounting for approximately $60 \%$ of deaths and one-third of national medical expenses. Therefore, the need for measures to deal with lifestyle-related diseases has been suggested [11]. Lifestyle-related diseases originate from unhealthy lifestyle habits causing obesity and insulin resistance, leading to the rising incidence of hypertension, diabetes, and dyslipidemia, and consequently the development of cardiovascular disease. Initial intervention is likely to succeed in disease prevention, but on the other hand, delayed intervention is likely to be less effective. Recently, there have been many reports on the association between SRH and lifestyle habits [12-23]. However, little is known about the relationship between SRH and lifestylerelated diseases or clinical parameters, which could be linking factors between SRH and lifestyle habits.

In the present study, significant increases in the prevalences of cancer and cardiovascular disease, treatment of lifestyle-related diseases, and abnormalities in clinical parameters were associated with lower SRH. Although the present study has a potential limitation because of its crosssectional nature, SRH shows a strong association with the 
prevalences of lifestyle-related diseases and abnormalities in clinical parameters. Thus, SRH is considered useful as a comprehensive indicator of lifestyle-related diseases for individual health management. SRH is also expected to be applicable to populational health management. Further investigations are necessary to evaluate whether SRH is useful for that purpose by analyzing the transition of SRH and objective health indicators in the same population as well as comparison among different populations.

The association of SRH with lifestyle habits was restricted; some dietary habits and heavy drinking did not show significant associations. Of the questions included in the present study, the results of weight gain (obesity) [13, 14, 16, 17], lack of exercise [12-17, 20, 22, 23], smoking [12-16, 18, 21, 22], and insufficient rest [23] were consistent with those of previous studies. Regarding diet, all Western studies asked about fruit and vegetable consumption and reported that those who consumed sufficient fruits and vegetables were likely to have better SRH [13, $15,16,22]$. In the present study, the pattern of eating behavior was examined, but the results cannot be simply compared with those of previous studies. As for drinking, several studies have reported that moderate alcohol consumption had a favorable effect on SRH, but the effect of nondrinking or heavy drinking on SRH has been controversial $[12,13,15,18,19,22]$. Thus, it is unreasonable to use SRH as a comprehensive indicator of lifestyle habits, and another indicator needs to be developed.

In conclusion, SRH is strongly associated with the prevalences of lifestyle-related diseases and abnormalities in clinical parameters. Therefore, SRH is considered useful as a comprehensive indicator of lifestyle-related health status. We are planning to validate the usefulness of SRH for evaluation of health status transition in a longitudinal study.

Acknowledgments This study was supported in part by Grants-inAid for Young Scientists (B) to C.Y. (22790493) from the Ministry of Education, Culture, Sports, Science, and Technology, Japan.

Conflict of interest The authors declare that they have no conflict of interest.

\section{References}

1. Mitoku K, Takahashi T, Hoshi T. Reviewing the studies of the relation between the subjective feeling of health and mortality. Kawasaki Med Welf J. 2006;16:1-10. (in Japanese).

2. Benjamins MR, Hummer RA, Eberstein IW, Nam CB. Selfreported health and adult mortality risk: an analysis of causespecific mortality. Soc Sci Med. 2004;59:1297-306.

3. Ministry of Health, Labour and Welfare. Standard program for specific health examinations and health guidance (final version). 2007 (in Japanese). http://www.niph.go.jp/soshiki/jinzai/ koroshoshiryo/kenshin/data/zentai.pdf. Accessed 11 Oct 2011.
4. Matthews DR, Hosker JP, Rudenski AS, Naylor BA, Treacher DF, Turner RC. Homeostasis model assessment: insulin resistance and beta-cell function from fasting plasma glucose and insulin concentrations in man. Diabetologia. 1985;28:412-9.

5. Yamada C, Mitsuhashi T, Hiratsuka N, Inabe F, Araida N, Takahashi E. Optimal reference interval for homeostasis model assessment of insulin resistance (HOMA-IR) in a Japanese population. J Diabetes Invest. 2011;2:373-6.

6. Mantel N. Chi-square tests with one degree of freedom; extensions of the Mantel-Haenszel procedure. J Am Stat Assoc. 1963;58:690-700.

7. Ministry of Health, Labour and Welfare. Comprehensive survey of living conditions. 2010 (in Japanese). http://www.e-stat.go.jp/ SG1/estat/GL08020103.do?_toGL08020103_\&listID=00000108 $3963 \&$ disp $=$ Other\&requestSender $=$ dsearch. Accessed 30 Jan 2012.

8. Suchman EA, Phillips BS, Streib GF. An analysis of the validity of health questionnaires. Soc Forces. 1958;36:223-32.

9. Friedsam HJ, Martin HW. A comparison of self and physician's ratings in an older population. J Health Hum Behav. 1963;4: 179-83.

10. Maddox GL, Douglass EB. Self-assessment of health: a longitudinal study of elderly subjects. J Health Soc Behav. 1973;14:87-93.

11. Takahashi E. Principle of the specific health examination and health guidance. In: Takahashi E, editor. Specific health guidance manual. Tokyo: Ishiyaku Publishers, Inc.; 2009. p. 28-41 (in Japanese).

12. Cott CA, Gignac MA, Badley EM. Determinants of self rated health for Canadians with chronic disease and disability. J Epidemiol Community Health. 1999;53:731-6.

13. Manderbacka K, Lundberg O, Martikainen P. Do risk factors and health behaviours contribute to self-ratings of health? Soc Sci Med. 1999;48:1713-20.

14. Svedberg P, Bardage C, Sandin S, Pedersen NL. A prospective study of health, life-style and psychosocial predictors of selfrated health. Eur J Epidemiol. 2006;21:767-76.

15. Harrington J, Perry IJ, Lutomski J, Fitzgerald AP, Shiely F, McGee $\mathrm{H}$, et al. Living longer and feeling better: healthy lifestyle, self-rated health, obesity and depression in Ireland. Eur J Public Health. 2010;20:91-5.

16. Kwaśniewska M, Kaleta D, Dziankowska-Zaborszczyk E, Drygas W, Makowiec-Dabrowska T. Lifestyle index and self-rated health status. Int J Occup Med Environ Health. 2007;20:349-56.

17. Molarius A, Berglund K, Eriksson C, Lambe M, Nordström E, Eriksson HG, et al. Socioeconomic conditions, lifestyle factors, and self-rated health among men and women in Sweden. Eur J Public Health. 2007;17:125-33.

18. Ford J, Spallek M, Dobson A. Self-rated health and a healthy lifestyle are the most important predictors of survival in elderly women. Age Ageing. 2008;37:194-200.

19. Powers JR, Young AF. Longitudinal analysis of alcohol consumption and health of middle-aged women in Australia. Addiction. 2008;103:424-32.

20. Södergren M, Sundquist J, Johansson SE, Sundquist K. Physical activity, exercise and self-rated health: a population-based study from Sweden. BMC Public Health. 2008;8:352.

21. Nakata A, Takahashi M, Swanson NG, Ikeda T, Hojou M. Active cigarette smoking, secondhand smoke exposure at work and home, and self-rated health. Public Health. 2009;123:650-6.

22. Tsai J, Ford ES, Li C, Zhao G, Pearson WS, Balluz LS. Multiple healthy behaviors and optimal self-rated health: findings from the 2007 Behavioral Risk Factor Surveillance System Survey. Prev Med. 2010;51:268-74.

23. Girón P. Determinants of self-rated health in Spain: differences by age groups for adults. Eur J Public Health. 2012;22(1):36-40. 